\title{
The Fundamental Theorem of Calculus in
}

\author{
$\mathbb{R}^{n}$ \\ Ulrich Mutze \\ www.ulrichmutze.de
}

It is shown that there is a more direct extension of the well-known Fundamental Theorem of Calculus (FTC) to the case of several variables than the one provided by the General Stokes' Theorem.

\section{The Theorem}

Let $C\left(\mathbb{R}^{n}\right)$ denote the set of continuous real-valued functions of $n$ real variables. For $f \in C\left(\mathbb{R}^{n}\right)$ and $a, b \in \mathbb{R}^{n}$ we define

$$
\int_{a}^{b} f(x) \mathrm{d} x:=\int_{a_{n}}^{b_{n}}\left(\ldots\left(\int_{a_{1}}^{b_{1}} f\left(x_{1}, \ldots, x_{n}\right) \mathrm{d} x_{1}\right) \ldots\right) \mathrm{d} x_{n},
$$

where it is not assumed that $a_{i} \leq b_{i}$ and it is understood that reversal of boundaries changes the sign of the integral. Further we define

$$
\frac{\mathrm{d}}{\mathrm{d} x} f(x):=f^{\prime}(x):=\frac{\partial}{\partial x_{1}} \cdots \frac{\partial}{\partial x_{n}} f\left(x_{1}, \ldots, x_{n}\right)
$$

if the objects on the right hand side (i.e. the limits involved) exist and depend on $x$ in such a way that $f^{\prime} \in C\left(\mathbb{R}^{n}\right)$. Let $\tilde{C}\left(\mathbb{R}^{n}\right)$ denote the set of $f \mathrm{~s}$ for which $f^{\prime}$ is defined in this sense. Finally we define that $F$ is an antiderivative of $f \in C\left(\mathbb{R}^{n}\right)$ iff $F \in \tilde{C}\left(\mathbb{R}^{n}\right)$ and $F^{\prime}=f$.

The First Fundamental Theorem of Calculus states that

$$
\int_{a}^{b} f(x) \mathrm{d} x=\left.F\right|_{a} ^{b}
$$

for all $a, b \in \mathbb{R}^{n}$, where $F$ is any antiderivative of $f$ and

$$
\left.F\right|_{a} ^{b}:=\sum_{\varepsilon_{1}, \ldots, \varepsilon_{n}=0}^{1}(-1)^{\varepsilon_{1}+\ldots+\varepsilon_{n}} F\left(\varepsilon_{1} a_{1}+\bar{\varepsilon}_{1} b_{1}, \ldots, \varepsilon_{n} a_{n}+\bar{\varepsilon}_{n} b_{n}\right), \quad \bar{\varepsilon}_{i}:=1-\varepsilon_{i} .
$$


This sum of $2^{n}$ terms reads for $n \in 1,2,3$ as follows

$$
\begin{aligned}
& F\left(b_{1}\right)-F\left(a_{1}\right), \\
& F\left(b_{1}, b_{2}\right)-F\left(b_{1}, a_{2}\right)-F\left(a_{1}, b_{2}\right)+F\left(a_{1}, a_{2}\right), \\
& F\left(b_{1}, b_{2}, b_{3}\right)-F\left(b_{1}, a_{2}, b_{3}\right)-F\left(a_{1}, b_{2}, b_{3}\right)+F\left(a_{1}, a_{2}, b_{3}\right) \\
& \quad-F\left(b_{1}, b_{2}, a_{3}\right)+F\left(b_{1}, a_{2}, a_{3}\right)+F\left(a_{1}, b_{2}, a_{3}\right)-F\left(a_{1}, a_{2}, a_{3}\right) .
\end{aligned}
$$

and is an alternating sum over values which $F$ takes at the $2^{n}$ vertexes of the domain of integration, which we simply call a rectangle, following Loomis and Sternberg [4], p. 324 ( an orthodox denomination would be axis-parallel rectangular parallelepiped).

The Second Fundamental Theorem of Calculus says that for any $a \in \mathbb{R}^{n}$

$$
F(x):=\int_{a}^{x} f(y) \mathrm{d} y
$$

defines an antiderivative $F$ of $f$ and that any antiderivative of $f$ deviates from $F$ at most by a sum of functions each which depending only on a strict subset of the $n$ variables under consideration.

The differential operator $\frac{\partial}{\partial x_{1}} \cdots \frac{\partial}{\partial x_{n}}$ is of first order with respect to each variable individually, so it is a very specific instance of a differential operator of order $n$. From the previously stated facts one obtains a formula for $f^{\prime}(x)^{1}$ which involves only a single limit and is also useful for numerical computation

$$
f^{\prime}(x)=\left.\lim _{h \rightarrow 0} \frac{1}{(2 h)^{n}} f\right|_{x-(h, \ldots, h)} ^{x+(h, \ldots, h)}
$$

\section{Discussion}

It is only a technical matter to proof these statements by induction, starting from their known truth for $n=1$. A more direct evidence comes from observing that the $n=1$ case implies all previous statements for functions of the type

$$
f\left(x_{1}, \ldots, x_{n}\right)=f_{1}\left(x_{1}\right) \cdots f_{n}\left(x_{n}\right)
$$

and, thus, for all finite linear combinations of such functions.

The present theorem contrasts with an opinion which is inherent in most teaching on calculus and which is comprehensively stated in

http:/mathforum.org/library/drmath/view/53755.html

as follows:

\footnotetext{
${ }^{1}$ Notice that this notation is introduced here to achieve uniformity with the one-dimensional case. It is not meant to replace the standard notion of a derivative which is about the best linear approximation of the function near a point. I thank Michael Livshits for having pointed out this potential source of misunderstanding.
} 
'In higher dimensions, there is no Fundamental Theorem of Calculus connecting multiple integrals with partial derivatives, so there isn't an "antidifferentiation" process for functions of several variables. The closest correspondence would probably [be?] the Divergence Theorem or Stokes Theorem, which connects integrals of certain ...

...Multiple integrals can often be evaluated as iterated (or repeated) onedimensional integrals so that the usual techniques can be used, but this doesn't always work.'

Most textbooks avoid such direct statements but create the same impression by their selection of material.

The reader will probably not escape the feeling that the stated theorem is nearly trivial: In a sense, the normal FTC is simply applied to repeated one-dimensional integrals (see the citation above). However, it is not completely trivial to find the notions that allow to give this 'repeated FTC' the form and logical structure of the FTC with $n$ as a variable. That this works suggests that the antiderivative in $n$ dimensions, as defined above, is a more natural construct than it is apparently recognized to be. For instance, it is at least a nice change in perspective do understand the area of a (planar) rectangle as resulting from the antiderivative $F(x, y)=x y$ of the constant function $f(x, y)=1$ via equation (4). Notice that $F$ can be selected as not containing redundant constants (although $F(x, y)=x y+137 x$ would also be a valid antiderivative of the constant function 1). The repeated integral approach, by contrast, would inject problem-related constants (edge coordinates of the rectangle) into the result of the first integration. If expressions are more complicated, such constants can create in the next integration step excessively complex expressions, for which today's computer algebra systems are unable to find the fitting simplifications. Especially, the programming-friendly property of (4) to represent the result by evaluations of the same function for varying values of the arguments will get lost in the proposed simplifications.

For a domain of integration which is a union of adjacent rectangles (a paved set in the sense of [4] p. 326, Definition 3.1) - which is a useful approximation in computational physics - equation (4), when applied to each of the rectangles, can easily be seen to express the integral of a function as a sum of evaluations of any antiderivative at surface points. So we have a simple connection between an integral of a function over such a domain and a sum of values that the antiderivative takes on the surface. This is an exact relation that could also be obtained from the General Stokes' Theorem. However, due to the edgy nature of our surface such an application could not be justified directly by the normal textbook version of the theorem which is for smooth surfaces.

It might be instructive to see at least the formal part of this connection. We use $n=$ 3 as a pattern for the general case: Let $f$ be the function to be integrated and $F$ an antiderivative of $f$. We consider the differential form

$$
\omega:=\frac{1}{3}\left[\left(\frac{\partial}{\partial x_{2}} \frac{\partial}{\partial x_{3}} F\right) \mathrm{d} x_{2} \wedge \mathrm{d} x_{3}+\left(\frac{\partial}{\partial x_{3}} \frac{\partial}{\partial x_{1}} F\right) \mathrm{d} x_{3} \wedge \mathrm{d} x_{1}+\left(\frac{\partial}{\partial x_{1}} \frac{\partial}{\partial x_{2}} F\right) \mathrm{d} x_{1} \wedge \mathrm{d} x_{2}\right]
$$


which obviously satisfies

$$
\mathrm{d} \omega=\left(\frac{\partial}{\partial x_{1}} \frac{\partial}{\partial x_{2}} \frac{\partial}{\partial x_{3}} F\right) \mathrm{d} x_{1} \wedge \mathrm{d} x_{2} \wedge \mathrm{d} x_{3} .
$$

Then the General Stokes' Theorem says for suitable subsets $\Omega \subset \mathbb{R}^{3}$ with surface $\partial \Omega$

$$
\begin{aligned}
\int_{\Omega} f\left(x_{1}, x_{2}, x_{3}\right) \mathrm{d} x_{1} \mathrm{~d} x_{2} \mathrm{~d} x_{3} & =\int_{\Omega}\left(\frac{\partial}{\partial x_{1}} \frac{\partial}{\partial x_{2}} \frac{\partial}{\partial x_{3}} F\left(x_{1}, x_{2}, x_{3}\right)\right) \mathrm{d} x_{1} \wedge \mathrm{d} x_{2} \wedge \mathrm{d} x_{3} \\
& =\int_{\Omega} \mathrm{d} \omega=\int_{\partial \Omega} \omega .
\end{aligned}
$$

If here $\Omega$ is a rectangle, the surface $\partial \Omega$ is the union of six two-dimensional rectangles. Due to the particular form of $\omega$, the integrals over these surface parts are can directly expressed in terms of values of $F$ by means of (3) for $n=2$. Adding the contributions from the six faces, we get the same result which gave (3) for $n=3$ directly.

It is natural that Stokes' Theorem gives the result not at once but iteratively since $\omega$ does not contain the 'full antiderivative $F^{\prime}$ but only antiderivatives with respect to a single variable: Let us choose functions $F^{1}, F^{2}, F^{3}$ such that $\frac{\partial}{\partial x_{1}} F^{1}=\frac{\partial}{\partial x_{2}} F^{2}=\frac{\partial}{\partial x_{3}} F^{3}=f$ then $\omega$ from (9) can also be written

$$
\omega:=\frac{1}{3}\left(F^{1} \mathrm{~d} x_{2} \wedge \mathrm{d} x_{3}+F^{2} \mathrm{~d} x_{3} \wedge \mathrm{d} x_{1}+F^{3} \mathrm{~d} x_{1} \wedge \mathrm{d} x_{2}\right) .
$$

Whereas the General Stokes' Formula can be viewed as performing one of the three integrations (by transforming a volume integral into a surface integral) the formulas (3), (4) do the whole integration at once, just as (7) defines a product of three differential operators by a single limit.

In all cases where it is feasible to compute $F$, it is even more feasible to compute $F^{1}, F^{2}, F^{3}$ and thus $\omega$. For sufficiently regular domains $\Omega$ such as spheres, this surface integral $\int_{\partial \Omega} \omega$ may be an advantageous representation of the original volume integral $\int_{\Omega} f \mathrm{~d} x_{1} \mathrm{~d} x_{2} \mathrm{~d} x_{3}$.

Formulas (5) - for sufficiently regular $\mathrm{F}$ - as a means to define a (not necessarily positive) measure are studied in [2], p. 205-207 and in a hidden manner in [1] p. 85. Both sources deal explicitly only with two variables. They then introduce, without mentioning the name, what is known as the Radon-Nikodym derivative of this measure with respect to the Lebesgue measure. Riesz calls this 'a derivative of a very particular type' and it is only in a well hidden statement that it is identified as 'the mixed second derivative of a function $f(x, y)^{\prime}$. Another connection to well-known facts can be found in [3], p. 3-4 in a study of the partial differential equation $u_{x y}=f(x, y)$.

I came to consider the matter by the practical need (for the work [5]) to have an efficient explicit formulas for the electrostatic potential of a rectangular patch of uniform surface charge. Here, the computer algebra system Mathematica allowed to find Integrate[Integrate[ $[1 / \mathrm{r}, \mathrm{x}], \mathrm{y}$ ] (for $\mathrm{r}:=\sqrt{\mathrm{x}^{2}+\mathrm{y}^{2}+\mathrm{z}^{2}}, \mathrm{z}$ is treated as a parameter here) and thus an antiderivative of $1 / \mathrm{r}$. Then the integral of the Coulomb potential over a rectangular region of the $x$-y-plane follows from the 'First FTC for 2 
variables' in a form that, due to the repetitive structure of equation (4), can be programmed compactly. That the required antiderivative can also be requested in the form Integrate[ $1 / r, x, y]$ (which seems to be an un-documented feature of Mathematica so far) shows that Wolfram Research is aware of the concept of an antiderivative with respect to several variables. Since there seems to be no automatic way for Mathematica to produce a purely real result for the antiderivative of $1 / \mathrm{r}$, one has to interfere manually which could introduce errors (see, however, the addition 2010-01-02). Here it helps that the correctness of an expression for the antiderivative can easily be checked by differentiation. See the figure entitled 'Now correct' in www.ulrichmutze.de/talks/ica1.pdf (1.2MB) for a pictorial representation of the electrostatic potential of a charge patch. The representation of the rectangular charge patch as a $\mathrm{C}++$ class 'PatchCharge' can be found in sections cpmforcesx.h and cpmforcescpp.h of www.ulrichmutze.de/softwaredescriptions/cpmlisting.pdf (3.7 MB).

Addition 2010-01-02: The above reported behaviour of Mathematica referred to version 6 of that program. Today, Mathematica 7 evaluates the expression Integrate $[1 / \mathrm{r}, \mathrm{x}, \mathrm{y}]$ virtually instantaneously as the real expression

$-x+z \operatorname{ArcTan}[x / z]-z \operatorname{ArcTan}\left[(x y) /\left(z \operatorname{Sqrt}\left[x^{\wedge} 2+y^{\wedge} 2+z^{\wedge} 2\right]\right)\right]+$

$y \log \left[2\left(x+\operatorname{Sqrt}\left[x^{\wedge} 2+y^{\wedge} 2+z^{\wedge} 2\right]\right)\right]+x \log \left[2\left(y+\operatorname{Sqrt}\left[x^{\wedge} 2+y^{\wedge} 2+z^{\wedge} 2\right]\right)\right]$

\section{Acknowledgment}

I thank Michael Livshits for a furthering discussion on the topic and Wolfgang Lücke for encouraging remarks.

\section{References}

[1] Frigyes Riesz and Béla Sz.-Nagy: Functional Analysis (Translated from the 2nd French edition) Dover 1990

[2] W.L. Smirnow: Lehrgang der Höheren Mathematik Teil V, Deutscher Verlag der Wissenschaften, Berlin 1962

[3] R. Courant and D. Hilbert: Methods of Mathematical Physics, Volume II Partial Differential Equations, Interscience Publishers, New York and London 1962

[4] Lynn H. Loomis and Shlomo Sternberg: Advanced Calculus, Addison-Wesley 1968

[5] U. Mutze, E. Stelter, T. Dera: Simulation of Electrophotographic Development, Final Program and Proceedings of IS\& T's NIP19: International Conference on Digital Printing Technologies September 28 - October 3, 2003 (IS\& T: The Society for Imaging Science and Technology, Springfield VA, 2003) p.57.

Last modification: 2010-01-02

Original (2004) at

www.ma.utexas.edu/mp_arc/c/04/04-165.pdf 